\title{
Contrast-enhanced ultrasound with a novel nanoparticle contrast agent for clinical diagnosis in patients with non-small cell lung cancer
}

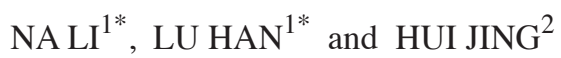 \\ ${ }^{1}$ Department of Ultrasound, General Hospital of Daqing Oilfield, Daqing, Heilongjiang 163000; \\ ${ }^{2}$ Department of Ultrasound, Harbin Medical University Cancer Hospital, Harbin, Heilongjiang 150040, P.R. China
}

Received March 9, 2016; Accepted March 3, 2017

DOI: $10.3892 /$ etm.2017.4933

\begin{abstract}
Non-small cell lung cancer (NSCLC) initiates in the non-small cells of the lung and is one of the most common types of human cancer. It is known for its rapid growth, easy migration, invasion and reoccurrence, and has the highest incidence rate of all types of cancer. Early detection of NSCLC is difficult to achieve and this is the primary reason for low survival rates in NSCLC. Contrast-enhanced ultrasound is the most common application for evaluating patients diagnosed with NSCLC. In the present study, a contrast-enhanced ultrasound combined with nanoparticles was performed with the aim of identifying patients with NSCLC at an early stage. The present study evaluated the effectiveness of administering a nanoparticle contrast agent through respiration in combination with enhanced ultrasound at improving image quality compared with traditional ultrasound. This maybe a novel method of detecting early-stage tumors in patients. There are numerous benefits to conducting diagnoses of NSCLC using contrast-enhanced ultrasound. It is a non-invasive imaging modality, induces little pain, has a low cost, an extensive range and produces high-resolution images. This means that it is safer and more beneficial to use in patients with NSCLC than conventional imaging examinations, including X-ray and bronchoscopy. Furthermore, the nanoscale microbubble contrast agent containing liposome-encapsulated epidermal growth factor receptor was inhaled by nebulization, which may lead to an enhanced ultrasound image. The sensitivity of contrast-enhanced ultrasound was investigated in mice with early stage NSCLC. The results indicated that ultrasound
\end{abstract}

Correspondence to: Professor Hui Jing, Department of Ultrasound, Harbin Medical University Cancer Hospital, 150 Haping Road, Harbin, Heilongjiang 150040, P.R. China

Email: huijingmedicine@163.com

${ }^{*}$ Contributed equally

Key words: non-small cell lung cancer, contrast-enhanced ultrasound, nanoscale microbubble administrated with nanoscale microbubble enhanced sensitivity and improved image quality compared with simple ultrasound. Furthermore, enhanced ultrasound resulted in a reliable and sensitive assessment of tumor mass in early-stage tumors. Altogether, contrast-enhanced ultrasound facilitated the efficient detection of NSCLC in patients in situ at an early stage. This protocol improved the understanding of the pathophysiology of NSCLC and may be applied in the detection of early-stage tumors in patients suspected of having cancer.

\section{Introduction}

Lung cancer is one of the most malignant types of cancer and has the fastest growing incidence of 25.9 in 100,000 individuals and mortality rates of 22.5 in 100,000 individuals among all cancers (1). Lung cancer is a respiratory disease with higher cancer-associated mortality compared with other types of cancer, due to worsening air conditions worldwide (2). Non-small cell lung cancer (NSCLC) is the major form present, which is initiated in the non-small cells of the lung and readily develops into malignant cancer that spreads throughout the body $(3,4)$. Previous studies have demonstrated that NSCLC has the highest incidence rate among all cancer types worldwide $(5,6)$. It is the most common type of lung cancer accounting for $>80 \%$ of all lung cancer cases and includes squamous cell carcinoma, large cell carcinoma and adenocarcinoma (7-9).

Although numerous studies have suggested therapeutic improvements in the treatment of NSCLC, the 5-year survival rate of patients remains very low at $<15 \%$, which is generally attributed to the high recurrent and metastatic rates (10-12). The main reason for the poor survival rate in patients is the rapid migration and aggressive invasion of NSCLC cells during comprehensive treatment and the high likelihood of recurrence $(13,14)$. Conventional treatments have little efficacy against NSCLC and frequent recurrence and metastasis often occurs in the clinic (14). In addition, a majority of patients with NSCLC are diagnosed with advanced stage lung cancer, which influenced the effectiveness of treatments due to increased apoptosis resistance and tumor metastasis (15). Therefore, identifying effective protocols to deliver an early diagnosis of NSCLC becomes a priority for researchers. 
Early diagnosis of patients with cancer is favorable for treatment, as it increases the survival time and decreases the pain and the economic burden experienced by patients with NSCLC (16). In the present study, a contrast-enhanced ultrasound combined with nanoparticle contrast agent was introduced, which could potentially be applied to improve imaging modality for NSCLC. This agent exhibited advantages for both early diagnosis and final confirmation of suspicious cases. In addition, it was concluded from previous studies that optimal therapeutic outcomes are dependent on early diagnosis and treatment $(16,17)$.

The mechanism of ultrasound diagnosis is dependent on short waves that penetrate soft tissue in order to identify the presence of any lumps. This is achieved in tissues by the bouncing back of waves when they reach the lumps (18). Contrast-enhanced ultrasound is currently used to diagnose cardiovascular conditions, as it is non-invasive, painless, relatively cheap, has an extensive range and high-resolution $(19,20)$. Notably, contrast-enhanced ultrasound is widely applied in the diagnosis of hypertrophic cardiomyopathy (21). Microbubbles are the most common used ultrasound contrast agent and provide a strongly reflective interface and resonate to ultrasound waves, which are easily observed (22). In the present study, an improved contrast-enhanced ultrasound administrated with a nanoparticle contrast agent aimed to improve image quality and resolution, thus improving the rate of early diagnosis of NSCLC compared with a standard ultrasound. This improved the ultrasound contrast and boosted the diagnostic accuracy in patients with NSCLC compared with standard ultrasound, which may have important clinical implications.

\section{Materials and methods}

Patients. A total of 302 patients with suspicious NSCLC were enrolled from Department of Ultrasound, General Hospital of Daqing Oilfield between January 2014 and January 2015 in the present study. The age range of patients was between 43 and 72 years old with a median age of 52 years old and the male (148) to female (154) ratio was approximately equal. Patients suspected of having NSCLC were eligible for the present study. Inclusion criteria was as follows: Those confirmed with NSCLC, with stage IV disease at the time of study entry, those with measurable disease at the time of study entry, those with resolution to grade $\leq 1$ adverse events, and those with adequate hematologic function, hepatic function, renal function and coagulation function. Exclusion criteria was as follows: Prior chemotherapy for relapsed and/or metastatic NSCLC, neo-adjuvant/adjuvant chemotherapy is permitted if at least 12 months has elapsed between the end of chemotherapy, prior treatment with Epidermal Growth Factor Receptor targeting small molecules or antibodies, radiotherapy or surgery (other than biopsy) within 4 weeks prior to enrollment, active brain metastases, any other current malignancy or malignancy diagnosed within the past 5 years, known pre-existing interstitial lung disease. The study protocol was approved by the Ethics Committee of General Hospital of Daqing Oilfield (QDGH: 2014061218; Daqing, China) and informed consent was obtained from all patients.
Principles and settings of contrast-enhanced ultrasound. The ultrasound diagnosis system analyzed contrast-enhanced ultrasound clinical trials using a preprogrammed setting. The preprogrammed setting was optimized to reach the best image formation. The mechanical index was set between 0.2-0.4 to avoid destruction of the fragile microbubbles that contain nanoparticles. The details of principles and settings of the contrast-enhanced ultrasound were as previously described (22).

Ultrasound contrast agents. A novel ultrasound contrast agent was introduced with the aim of diagnosing NSCLC at an early stage. The multi-targeted tyrosine kinase inhibitor Lenvatinib (Selleck Chemicals LLC, Boston, MA, USA) was covalently bound with the nanoparticles of super paramagnetic iron oxide particles as previously described (23). The nanoparticle ultrasound contrast agent and Optison (GE Healthcare Life Sciences, Shanghai, China) entered the lung by breathing and an atomizer (GE Healthcare Life Sciences) was used to ensure the entire lung was covered. Following administration by respiration, the nanoparticle ultrasound contrast agent was distributed through the respiratory system. The microbubbles, within the nanoparticle, contained the multi-targeted tyrosine kinase inhibitor Lenvatinib and Lenvatinib was capable of transporting the target to tumor cells. Furthermore, the microbubbles targeted the lesion through the pulmonary circulation due to their small diameter. After $30 \mathrm{~min}$, the nanoparticle ultrasound contrast agent could be visualized using an imaging system (General Electric GE Voluson S8; GE Healthcare Life Sciences). No side effects were observed in patients following its use.

Immunofluorescence and histological staining. Tumor cells were obtained from patients with NSCLC. The cells $\left(1 \times 10^{5}\right)$ were cultured for 72 at $37^{\circ} \mathrm{C}$ in 6-well plates in Dulbecco's modified Eagle's minimal essential medium (Gibco; Thermo Fisher Scientific, Inc., Waltham, MA, USA) supplemented with $10 \%$ heat-inactivated fetal bovine serum (Biowhittaker; Lonza, Basel, Switzerland). The cell density was adjusted to $5 \times 10^{4} / \mathrm{ml}$. The cells were then incubated with primary anti-carcino embryonic antigen (catalogue no. ab4451; Abcam, Cambridge, MA, USA) or PBS (mock group) for $12 \mathrm{~h}$ at $4^{\circ} \mathrm{C}$, then incubated with anti-rabbit immunoglobulin (Ig) $\mathrm{G}$ and anti-mouse IgG (catalogue nos. W4011 and W4021, respectively; Promega Corporation, Madison, WI, USA) for $2 \mathrm{~h}$ at $37^{\circ} \mathrm{C}$ at a dilution of 1:50 for squamous cell carcinoma antigen. Subsequently, cells were washed three times with PBS to remove the primary antibody at a dilution of 1:50 and the anti-goat IgG-horseradish peroxidase secondary antibody (catalogue no. A5420, Sigma-Aldrich; Merck KGaA, Darmstadt, Germany) at a dilution of 1:50 was added for $15 \mathrm{~min}$ at $4^{\circ} \mathrm{C}$. The cells were then washed with PBS to completely remove the secondary antibody. Finally, cells were observed using a fluorescence microscope. The cells were fixed in neutral formalin for $15 \mathrm{~min}$ at $37^{\circ} \mathrm{C}$ and then embedded in paraffin and tissue sections were cut to $4 \mu \mathrm{m}$ thick. Cells were then fixed with pre-warmed PBS $+4 \%$ paraformaldehyde for $30 \mathrm{~min}$ at $37^{\circ} \mathrm{C}$ and blocked with $\mathrm{PBS}+0.3 \%$ bovine serum albumin (BSA, Sigma-Aldrich; Merck KGaA) for $30 \mathrm{~min}$ at $30^{\circ} \mathrm{C}$. Conjugates [IgG-680-PTX (JZ200505), $\alpha$-CEA-680 
(JZ21834), and $\alpha$-CEA-680-PTX (XT200514), Thermo Fisher Scientific, Inc.] were serially diluted 1:2 $(60 \mu \mathrm{g} / \mathrm{ml})$ in PBS $+0.3 \%$ BSA and $1.62 \mu \mathrm{M}$ Hoechst 33342 dye (catalogue no. 62249; Thermo Fisher Scientific, Inc.) and incubated in 6 -well plates for $1 \mathrm{~h}$ at room temperature. Immunofluorescence procedures were performed as previously reported (24). For histological staining, tumor sections were stained with hematoxylin and eosin and observed under light microscope, as previously reported (25).

\section{Results}

Patient characteristics. A total of 302 patients with suspected NSCLC were enrolled in the test to confirm a final diagnosis of lung cancer. The mean age of the patients was $57.5 \pm 14.4$ years old and there were fewer male patients than the female ones. The primary details of the characteristics of the patients are summarized (Table I). The tumor cells did not appear to show tumor metastasis in all patients, as observed by the modified contrast-enhanced ultrasound. Among these patients, 42 out of 302 (13.9\%) suspicious NSCLC patients were diagnosed as tumor-free following analysis with contrast-enhanced ultrasound and subsequently confirmed by histological observation. Improved contrast-enhanced ultrasound demonstrated that $26.2 \%$ of patients $(79 / 302)$ were diagnosed with squamous cell carcinoma, $28.8 \%$ of patients $(87 / 302)$ were diagnosed with large cell carcinoma and $31.1 \%$ of patients (94/302) were diagnosed with adenocarcinoma according to the American Collegeof Chest Physicians evidence-based clinical practice guidelines (26). The diagnosis of NSCLC classification was summarized in Table II. These data indicated that the improved contrast-enhanced ultrasound of the present study was effective at diagnosing NSCLC.

Contrast-enhanced ultrasound: Squamous cell carcinoma. Primary squamous cell carcinoma of the lung is a common type of lung cancer (26). Although ultrasound detection has many benefits in the diagnosis of cancer, the effectiveness of ultrasound in the diagnosis of cancer is limited, particularly for rough, irregular shaped tumors and heterogeneous tumor stroma. In the present study, a contrast-enhanced ultrasound was used to diagnose early stage squamous cell carcinoma. According to the imaging findings (Fig. 1A), 26.2\% (79/302) of patients suspected of having NSCLC were diagnosed with squamous cell carcinoma at the primary sites of lung lesions (Table II). This diagnosis was confirmed by immunofluorescence and histological staining of squamous cell carcinoma (Fig. 1B). The observations of the present study exhibited high efficacy of the improved contrast-enhanced ultrasound combined with nanoparticle contrast agent in the diagnosis of squamous cell carcinoma, and all cases were consistent with the histological findings.

Contrast-enhanced ultrasound: Large cell carcinoma. A previous study (8) has indicated that large cell carcinoma accounts for $<10 \%$ in patients with lung cancer. Diagnosis of large cell carcinoma in the chest wall is challenging due to poor imaging quality of conventional ultrasound and the fact that features remain nonspecific in diagnostics. An accurate diagnosis in the clinic may provide an opportunity for
Table I. Patient characteristics.

\begin{tabular}{lcc}
\hline Characteristic & Males & Females \\
\hline Number & 148 & 154 \\
Age (years) & $45-72$ & $42-68$ \\
Medical history of cancer & 7 & 12 \\
Mean blood pressure (mmHg) & $124 \pm 10.8$ & $117 \pm 12.2$ \\
Mean blood glucose (mmol/l) & $5.3 \pm 3.4$ & $7.5 \pm 2.7$ \\
Smoker & 42 & 26 \\
Non-smoker & 23 & 72 \\
Ex-smoker & 44 & 93 \\
\hline
\end{tabular}

Table II. Diagnosis of 302 patients with non-small cell lung cancer, using enhanced-contrast ultrasound.

\begin{tabular}{lcc}
\hline Diagnosis, $\mathrm{n}(\%)$ & Males & Females \\
\hline Healthy & $22(52.4)$ & $20(47.6)$ \\
Squamous cell carcinoma & $30(11.5)$ & $49(18.8)$ \\
Large cell carcinoma & $32(12.3)$ & $55(21.2)$ \\
Adenocarcinoma & $27(10.4)$ & $67(25.8)$ \\
\hline
\end{tabular}

tumor eradication and complete rehabilitation. In the present study, the effectiveness of an improved contrast-enhanced ultrasound combined with a nanoparticle contrast agent at detecting early-stage large cell carcinoma was investigated. The diagnostic results indicated that $28.8 \%$ (87/302) of patients suspected of having NSCLC were diagnosed with large cell carcinoma (Table II). In addition, the diagnosis was further confirmed by immunofluorescence and histological staining of the large cell carcinoma in all patients with large cell carcinoma (Fig. 2A and B). These observations indicate that a contrast-enhanced ultrasound may be an efficient and inexpensive way to detect early stage large cell carcinoma.

Contrast-enhanced ultrasound: Adenocarcinoma. Primary adenocarcinoma of the lung tumors is one of the most common types of tumor, and this has been reported in previous study (4). Currently, contrast-enhanced ultrasonography is a potential and helpful medical protocol that may be used as a pain-free and non-invasive method of diagnosing adenocarcinoma. A previous study has documented the diagnosis of hepatocellular and cholangiocellular carcinomas or metastatic adenocarcinoma of the liver using contrast-enhanced ultrasonography and demonstrated contrast-enhanced ultrasonography was efficient at diagnosing tumors of the liver (27). However, to the best of our knowledge, there have been no studies analyzing the effectiveness of contrast-enhanced ultrasound used in the diagnosis of early-stage primary adenocarcinoma. The contrast-enhanced ultrasound data indicated that $31.1 \%$ (94/302) of patients with suspected NSCLC were confirmed to have early-stage adenocarcinoma by pathological analysis (Table II). The presence of adenocarcinomas in all patients was confirmed by immunofluorescence and histological staining (Fig. 3). Therefore, the results of contrast-enhanced ultrasound diagnosis of NSCLC 
B

A

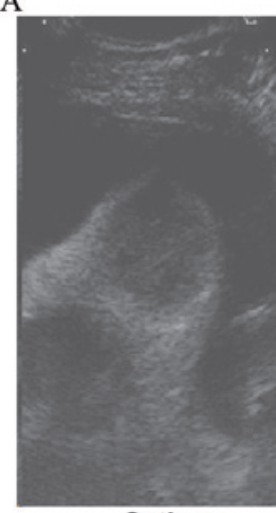

Optison



Napuca

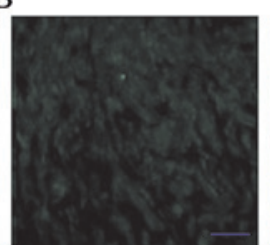

Mock

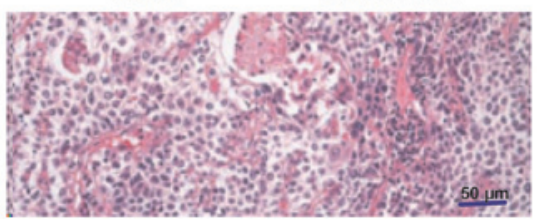

H\&E staining

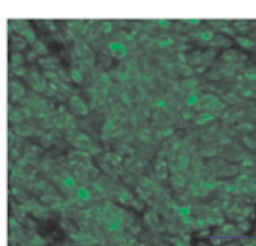

Tumor cells

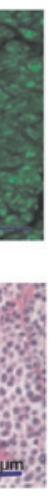

Figure 1. Squamous cell carcinoma diagnosis by contrast-enhanced ultrasound, immunofluorescence and histological staining. (A) Squamous cell carcinoma diagnosis by contrast-enhanced ultrasound with Optison or Napuca contrast agent. (B) Immunofluorescence and histological staining confirmation of patients with non-small cell lung carcinoma. Mock, no primary antibody addition; Arrow, location of squamous cell carcinoma.




Mock

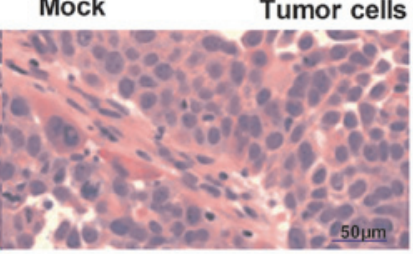

H\&E staining

Figure 2. Large cell carcinoma diagnosis by contrast-enhanced ultrasound, immunofluorescence and histological staining. (A) Large cell carcinoma diagnosis by contrast-enhanced ultrasound with Optison or Napuca contrast agent. (B) Immunofluorescence and histological staining confirmation of patients with large cell carcinoma. Mock, no primary antibody addition; Arrow, location of large cell carcinoma; box, range of adenocarcinoma.

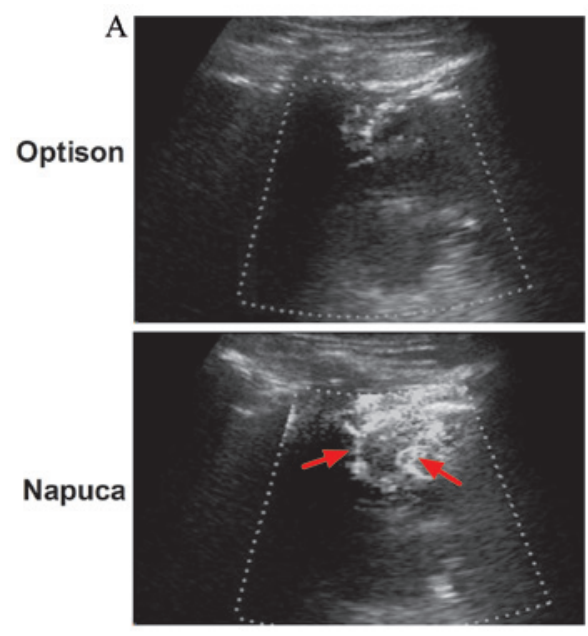

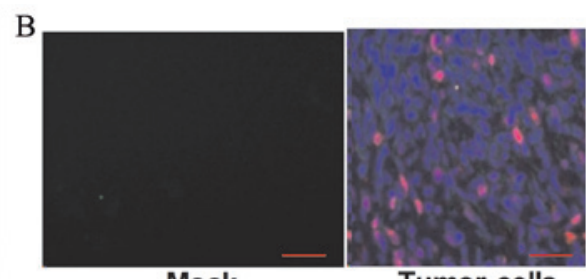

Mock Tumor cells



H\&E staining

Figure 3. Adenocarcinoma diagnosis by contrast-enhanced ultrasound, immunofluorescence and histological staining. (A) Adenocarcinoma diagnosis by contrast-enhanced ultrasound with Optison or Napuca contrast agent. (B) Immunofluorescence and histological staining confirmation of patients with adenocarcinoma. Mock, no primary antibody addition; Arrow, location of adenocarcinoma.

patients were consistent with the immunofluorescence and histological staining results, indicating that contrast-enhanced ultrasound combined with nanoparticle contrast agent is a reliable protocol and effective at diagnosing adenocarcinomas. 


\section{Discussion}

Lung cancer has the highest mortality rate out of all cancer types (28), including breast, prostate, colon and pancreas cancer (1). The number of lung cancer cases is increasing; there are $\sim 120$ million newly diagnosed cases of lung cancer each year. It has also been suggested that lung tumors are becoming more common in younger patients (29). Currently, the most efficient methods of treating lung cancer are surgery, radiotherapy, chemotherapy and immunotherapy (30). However, a majority $(>67 \%)$ of newly diagnosed patients are diagnosed at an advanced stage of cancer (31). Failure to identify the site of lung cancer severely delays the treatment of lung cancer. Furthermore, advanced stage lung cancer often exhibits drug resistance, and a majority of patients undergoing treatment experience side effects, such as vomiting and nausea (32). Therefore, lung cancer diagnosis protocols must be developed in order to make definite diagnoses.

Currently, five imaging protocols are used to examine and diagnose lung cancer in the clinic, including X-ray, computed tomography (CT) scanning, magnetic resonance imaging (MRI), positron emission tomography (PET) scanning and ultrasound. Among these auxiliary diagnostic methods, ultrasound is the most commonly used and induces minimum harm to the body (32). Furthermore, it is a non-invasive imaging modality, induces no pain, is low-cost, has an extensive range and high-resolution (33). Although ultrasound diagnosis is beneficial for use in the clinic, it has been previously reported that numerous factors, including performance status, comorbidity, sex and age, affect the diagnosis of patients with early-stage lung cancer (8). However, all of these factors cannot be modified prior to clinical application. Importantly, the early detection of lung cancer in patients has many benefits, including the increased likelihood of tumor eradication and patient survival (12).

The early diagnosis of NSCLC is a challenge that remains to be resolved in clinical practice. In the present study, contrast-enhanced ultrasound combined with a nanoparticle contrast agent was used to diagnose patients suspected of having NSCLC, and the site of lung cancer was also identified at an early stage. The improved contrast-enhanced ultrasound combined with nanoparticle contrast agent may not only have potential applications in patients with lung cancer, but may also be used in the diagnosis of other human diseases. Schinkel et al (22) reported that contrast-enhanced ultrasound may have clinical applications in patients with atherosclerosis and the study indicated that new developments in contrast-enhanced ultrasound-based molecular imaging may improve understanding of the pathophysiology of other diseases. This may facilitate the imaging and direct diagnosis of varying types of cancers in the future.

In conclusion, the present study indicated that contrast-enhanced ultrasound improved the imaging quality and diagnostic accuracy of early-stage NSCLC. The use of an improved contrast-enhanced ultrasound combined with a nanoparticle contrast agent did not induce any short-term harm and nanoparticles were absorbable without affecting the respiratory system. In addition, the ultrasound nanoparticle contrast agent has the potential for use in cancer target therapy by taking advantage of the therapeutic capabilities of anticancer agents. The contrast-enhanced ultrasound combined with nanoparticles contrast agent used to assess patients with NSCLC may therefore be developed to diagnose other types of cancer in humans.

\section{References}

1. Bray F, Jemal A, Grey N, Ferlay J and Forman D: Global cancer transitionsaccording to the Human Development Index (2008-2030): A population-based study. Lancet Oncol 13: 790-801, 2012

2. Fenton-Ambrose L and Kazerooni EA: Preventative care: Lung-cancer screens now worth the cost. Nature 514: 35, 2014.

3. Kong R, Feng J, Ma Y, Zhou B, Li S, Zhang W, Jiang J, Zhang J, Qiao Z, Zhang T, et al: Silencing NACK by siRNA inhibits tumorigenesis in non-small cell lung cancer via targeting Notch1 signaling pathway. Oncol Rep 35: 2306-2314, 2016.

4. Jiang SY, Zhao J, Wang MZ, Huo Z, Zhang J, Zhong W and $\mathrm{Xu}$ Y: Small-cell lung cancer transformation in patients with pulmonary adenocarcinoma: A case report and review of literature. Medicine (Baltimore) 95: e2752, 2016.

5. Tsim S, O'Dowd CA, Milroy R and Davidson S: Staging of non-small cell lung cancer (NSCLC): A review. Respir Med 104: $1767-1774,2010$

6. van Meerbeeck JP and Surmont VF: Stage IIIA-N2 NSCLC: A review of its treatment approaches and future developments. Lung Cancer 65: 257-267, 2009.

7. Brody H: Lung cancer. Nature 513: S1, 2014.

8. Moro-SibilotD,SmitE, de Castro CarpeñoJ,Lesniewski-Kmak K, Aerts JG, Villatoro R, Kraaij K, Nacerddine K, Dyachkova Y, Smith KT, et al: Non-small cell lung cancer patients with brain metastases treated with first-line platinum-doublet chemotherapy: Analysis from the European FRAME study. Lung Cancer 90: 427-432, 2015.

9. Barnett SA, Downey RJ, Zheng J, Plourde G, Shen R, Chaft J, Akhurst T, Park BJ and Rusch VW: Utility of routine PET imaging to predict response and survival after induction therapy for non-small cell lung cancer. Ann Thorac Surg 101: 1052-1059, 2016.

10. Voulgaris E, Pentheroudakis G, Vassou A and Pavlidis N: Disseminated intravascular coagulation (DIC) and non-small cell lung cancer (NSCLC): Report of a case and review of the literature. Lung Cancer 64: 247-249, 2009.

11. Kosmidis P: Chemotherapy in NSCLC: Historical review. Lung Cancer 38 (Suppl 3): S19-S22, 2002.

12. Stewart EL, Tan SZ, Liu G and Tsao MS: Known and putative mechanisms of resistance to EGFR targeted therapies in NSCLC patients with EGFR mutations-a review. Transl Lung Cancer Res 4: 67-81, 2015.

13. Müller B,Bovet M, Yin Y,StichelD,MalzM,González-Vallinas M, Middleton A, Ehemann V, Schmitt J, Muley T, et al: Concomitant expression of far upstream element (FUSE) binding protein (FBP) interacting repressor (FIR) and its splice variants induce migration and invasion of non-small cell lung cancer (NSCLC) cells. J Pathol 237: 390-401, 2015.

14. Zhao Q, Yue J, Zhang C, Gu X, Chen $\mathrm{H}$ and Xu L: Inactivation of $\mathrm{M} 2 \mathrm{AChR} / \mathrm{NF}-\kappa \mathrm{B}$ signaling axis reverses epithelial-mesenchymal transition (EMT) and suppresses migration and invasion in non-small cell lung cancer (NSCLC). Oncotarget 6: 29335-29346, 2015.

15. Kim DS, Park KM, Won YS, Kim JY, Lee JK, Kim JG, Oh ST, Jung SS and Kang WK: Occurrence and prognosis of symptomatic venous thromboembolism in colorectal cancer surgery patients. Vasc Specialist Int 30: 49-55, 2014.

16. Thunnissen E, Kerr KM, Herth FJ, Lantuejoul S, Papotti M, Rintoul RC, Rossi G, Skov BG, Weynand B, Bubendorf L, et al: The challenge of NSCLC diagnosis and predictive analysis on small samples. Practical approach of a working group. Lung Cancer 76: 1-18, 2012.

17. Herth F, Kirsch CM and Stoelben E: Diagnosis of non-small-cell lung carcinoma (NSCLC). Onkologie 29 (Suppl 2): S3-S6, 2006 (In German).

18. Kolisnichenko MM, Pykov MI and Poddubny IV: Ultrasonic diagnosis of girshprung illness at children. Eksp Klin Gastroenterol: 38-43, 2015 (In Russian).

19. Barzilay E, Harel Y, Haas J, Berkenstadt M, Katorza E, Achiron R and Gilboa Y: Prenatal diagnosis of amniotic band syndrome-risk factors and ultrasonic signs. J Matern Fetal Neonatal Med 28: 281-283, 2015. 
20. Isaev GB, Manafov SS and Alieva NZ: Ultrasonic diagnosis of functional and organic changes of colon in patients suffering from chronic constipation. Georgian Med News: 41-46, 2013 (In Russian).

21. Krylova NS, Demkina AE, Poteshkina NG and Khashieva FM: Tissue Doppler imaging and ultrasonic methods for evaluating myocardial deformation in the diagnosis of hypertrophic cardiomyopathy. Kardiologiia 54: 79-84, 2014 (In Russian).

22. Schinkel AF, Kaspar M and Staub D: Contrast-enhanced ultrasound: Clinical applications in patients with atherosclerosis. Int J Cardiovasc Imaging 32: 35-48, 2016.

23. Chen CL, Hu GY, Mei Q, Qiu H, Long GX and Hu GQ: Epidermal growth factor receptor-targeted ultra-small superparamagnetic iron oxide particles for magnetic resonance molecular imaging of lung cancer cells in vitro. Chin Med J (Engl) 125: 2322-2328, 2012.

24. Dirani M, Nasreddine W, Abdulla F and Beydoun A: Seizure control and improvement of neurological dysfunction in Lafora disease with perampanel. Epilepsy Behav Case Rep 2: 164-166, 2014.

25. Kargahi N, Razavi SM, Deyhimi P and Homayouni S: Comparative evaluation of eosinophils in normal mucosa, dysplastic mucosa and oral squamous cell carcinoma with hematoxylin-eosin, Congo red and EMR1 immunohistochemical staining techniques. Electron Physician 7: 1019-1026, 2015.

26. Lewis SZ, Diekemper R and Addrizzo-Harris DJ: Methodology for development of guidelines for lung cancer: Diagnosis and management of lung cancer, 3rd ed: American College of Chest Physicians evidence-based clinical practice guidelines. Chest 143 (5 Suppl): 41S-50S, 2013.
27. Nishigaki Y, Hayashi H, Tomita E, Suzuki Y, Watanabe N, Watanabe S, Watanabe C, Takagi Y, Kato T and Naiki T: Usefulness of contrast-enhanced ultrasonography using Sonazoid for the assessment of therapeutic response to percutaneous radiofrequency ablation for hepatocellular carcinoma. Hepatol Res 45: 432-440, 2015.

28. van der Wekken AJ, Saber A, Hiltermann TJ, Kok K, van den Berg A and Groen HJ: Resistance mechanisms after tyrosine kinase inhibitors afatinib and crizotinib in non-small cell lung cancer, a review of the literature. Crit Rev Oncol Hematol 100: 107-116, 2016.

29. Thill PG, Goswami P, Berchem G and Domon B: Lung cancer statistics in Luxembourg from 1981 to 2008. Bull Soc Sci Med Grand Duche Luxemb: 43-55, 2011.

30. Awad R and Nott L: Radiation recall pneumonitis induced by erlotinib after palliative thoracic radiotherapy for lung cancer: Case report and literature review. Asia Pac J Clin Oncol 12: 91-95, 2016.

31. Eton DT, Yost KJ and Cella D: Future trends in patient-reported outcomes assessment for patients with advanced-stage lung cancer receiving targeted therapy. Clin Lung Cancer 8: 99-109, 2006.

32. Soldan K, Pooley FD, Hansen J, Andersen A, Chang-Claude J, Ferro G, Ohgaki H, Skov BG, Cherrie JW, Saracci R and Boffetta P: Lung fibre burden in lung cancer cases employed in the rock and slag wool industry. Ann Occup Hyg 50: 241-248, 2006.

33. Condón-Abanto S, Arroyo C, Álvarez I, Condón S and Lyng JG: Application of ultrasound in combination with heat and pressure for the inactivation of spore forming bacteria isolated from edible crab (Cancer pagurus). Int J Food Microbiol 223: 9-16, 2016. 